\title{
Rasburicase-induced hemolytic anemia and methemoglobinemia in a patient with glucose-6- phosphate dehydrogenase deficiency.
}

\author{
Debergh C. MD, Dierickx D. MD PhD, Jannsens A. MD PhD, Vandenberghe P. MD PhD,
} UZ Leuven, Department of Hematology, Leuven, Belgium

\section{Background}

Glucose-6-phosphate dehydrogenase (G6PD) deficiency is an X-linked hereditary genetic defect due to mutations in the G6PD gene which cause functional variants with many clinical phenotypes. The most frequent manifestations of G6PD deficiency are neonatal jaundice and acute hemolytic anemia, which is usually triggered by an exogenous agent, such as rasburicase $(1,2)$.

\section{Case report}

- 46-year old woman

- Medical history: CLL since 2013

- Admission because of a large cell lymphoma

transformation

- Therapy with R-CHOP and rasburicase to prevent TLS

- Two days later: development of dyspnea, hypoxia, headache and general weakness

- Clinical examination: mild icterus and dark urine

- Laboratory findings : Hb $6 \mathrm{~g} / \mathrm{dl}$, trombopenia (40x 10^9/L).

DAT negative. Peripheral blood smear: strong excess of

fragmentocytes.

- Arterial blood gas: MetHb 5.3\%

- Patient confirms an intolerance for beans

A glucose-6-phosphate deficiency was suspected.

Therapy with rasburicase was stopped immediately. Following blood transfusion, oxygen supply and fluid administration the patient made a full recovery.

\section{Evolution of laboratory findings}

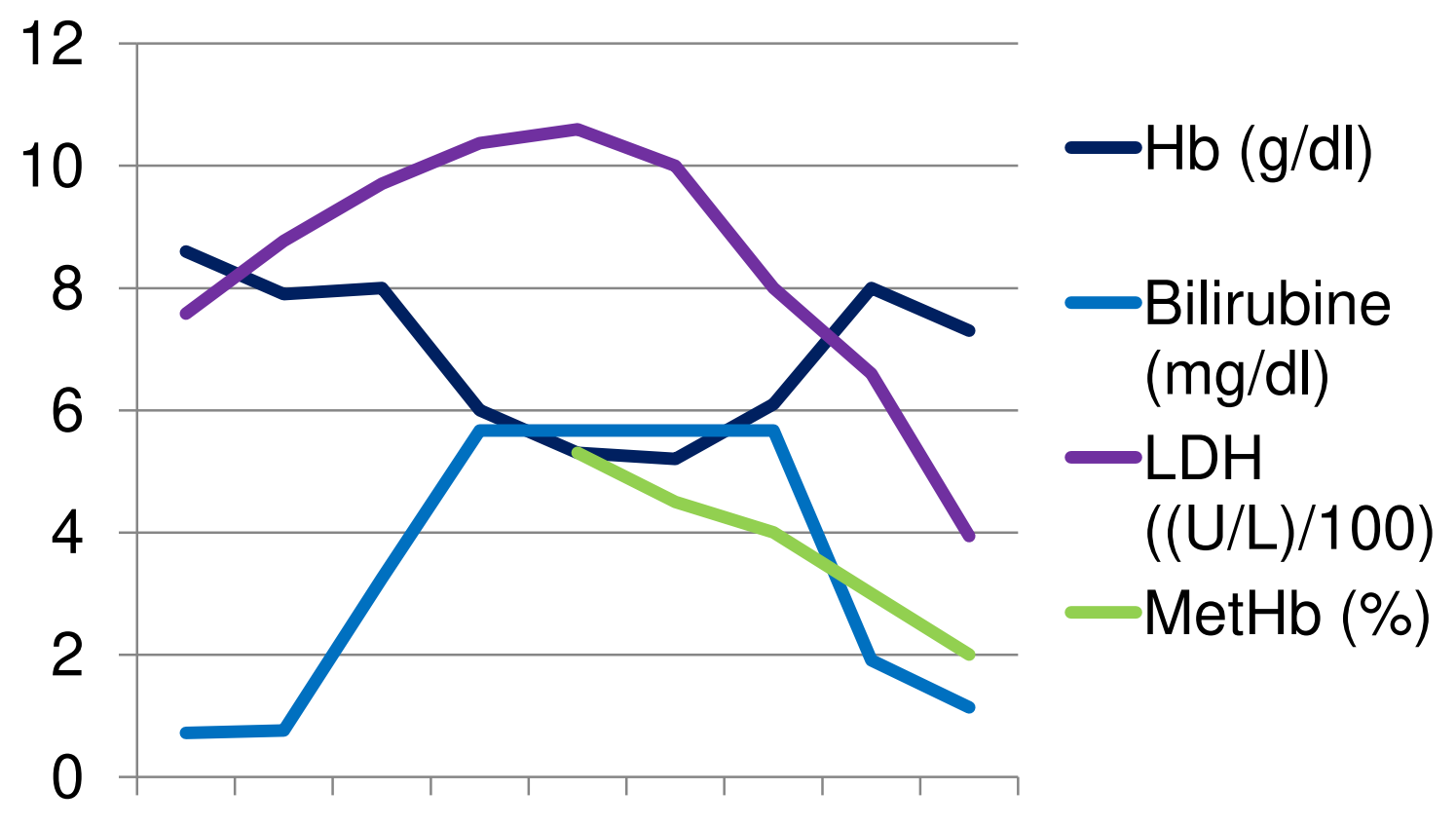

References:

1. Cappellini MD, Fiorelli G. Glucose-6-phosphate dehydrogenase deficiency. Lancet 2008 Jan 5;371(9606):64-74.

2. Phillpotts S, Tash E, Sen S. Glucose-6-phosphate dehydrogenase deficiency: an unusual cause of acute jaundice after paracetamol overdose. Eur J Haematol 2014 Nov;93(5):446-8. 3. $\mathrm{Ng} \mathrm{JS1}$, Edwards EM, Egelund TA. Methemoglobinemia induced by rasburicase in a pediatric patient: a case report and literature review. J Oncol Pharm Pract. 2012 Dec;18(4):425-31.

\section{Discussion}

Rasburicase is frequently used in patients with hematologic malignancies for tumor lysis syndrome (TLS) prophylaxis and therapy. However, as a result of the defects in the physiological antioxidant pathway, erythrocytes of patients with G6PD deficiency are not protected against the oxidating stress exerted by hydrogen peroxide generated with the administration of rasburicase (3). This results in acute hemolytic anemia and/or methemoglobinemia. To prevent this potentially life-threatening complication patients at risk for a G6PDdeficiency, based on ethnicity, should be screened prior to administration of chemotherapy. When hemolysis occurs, the most effective management consists of supportive therapy with transfusion and oxygen supply.

\section{Pentose Phophate pathway}

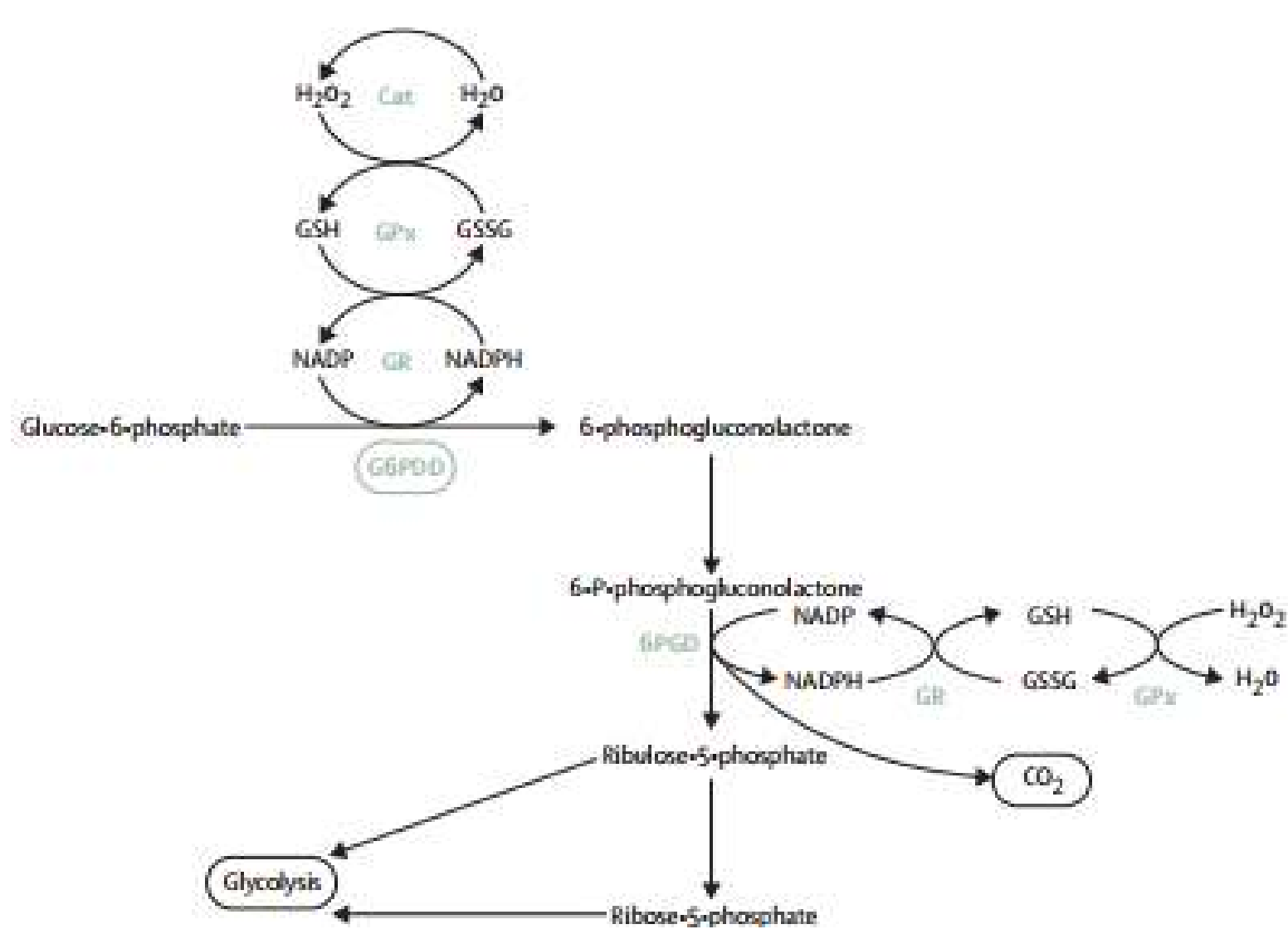

\section{Conclusion}

Patients from ethnicities in which a G6PD deficiency is prevalent should be screened prior to chemotherapy administration whenever possible. Therapy consists of packed red cell transfusion, oxygen supply and monitoring. 